\title{
Diagnostic testing for uniparental disomy: a points to consider statement from the American College of Medical Genetics and Genomics (ACMG)
}

\author{
Daniela del Gaudio, $\mathrm{PhD}^{1}$, Marwan Shinawi, $\mathrm{MD}^{2}$, Caroline Astbury, $\mathrm{PhD}^{3}$, Marwan K. Tayeh, $\mathrm{PhD}^{4}$, \\ Kristen L. Deak, $\mathrm{PhD}^{5}$ and Gordana Raca, MD, PhD ${ }^{6}$, ACMG Laboratory Quality Assurance Committee ${ }^{7}$
}

\begin{abstract}
Disclaimer: This statement is designed primarily as an educational resource for medical geneticists and other clinicians to help them provide quality medical services. Adherence to this statement is completely voluntary and does not necessarily assure a successful medical outcome. This statement should not be considered inclusive of all proper procedures and tests or exclusive of other procedures and tests that are reasonably directed to obtaining the same results. In determining the propriety of any specific procedure or test, the clinician should apply his or her own professional judgment to the specific clinical circumstances presented by the individual patient or specimen.

Clinicians are encouraged to document the reasons for the use of a particular procedure or test, whether or not it is in conformance with this statement. Clinicians also are advised to take notice of the date this statement was adopted, and to consider other medical and scientific information that becomes available after that date. It also would be prudent to consider whether intellectual property interests may restrict the performance of certain tests and other procedures.
\end{abstract}

Keywords: genomic imprinting; uniparental disomy; postnatal testing; prenatal testing
Genetics in Medicine (2020) 22:1133-1141; https://doi.org/10.1038/s41436020-0782-9

\section{OVERVIEW}

In 1980, Eric Engel ${ }^{1}$ first proposed the concept of uniparental disomy (UPD), in which both homologous chromosomes are inherited from one parent, with no contribution (for that chromosome) from the other parent. In 1988, the first case of a Mendelian disorder associated with UPD was reported, in which a child with cystic fibrosis (MIM 219700) had inherited two copies of a pathogenic variant in CFTR (MIM 602421) from a heterozygous carrier mother, with no contribution from the biological father. ${ }^{2}$

For the majority of chromosomes, UPD is without clinical consequence. However, for chromosomes $6,7,11,14,15$, and 20 , there are parent-of-origin or imprinting differences in gene expression in the context of UPD, which may lead to phenotypic abnormalities. In addition, UPD may uncover an autosomal recessive disorder on a chromosome that is not subject to imprinting, while UPD of the X chromosome may lead to X-linked recessive disorders in females. Rarely, inheritance of both sex chromosomes from the father, may result in father-to-son transmission of X-linked conditions. ${ }^{3}$

\section{MECHANISMS AND CLINICAL CONSEQUENCES OF UPD}

UPD generally results from two nondisjunction events, with the first event occurring during meiosis and the second being a mitotic event. Nondisjunction in meiosis I constitutes a failure of two homologues to segregate, which may eventually give rise to the presence of two different homologues from the same parent or heterodisomy. Nondisjunction in meiosis II is a failure of sister chromatids to separate into daughter cells, which subsequently can result in isodisomy. The gametes resulting from meiosis complicated by nondisjunction may be disomic (containing two copies of the affected chromosome) or nullisomic (containing no copies of the affected chromosome). Following fertilization with a normal haploid gamete, the zygote is expected to have either a trisomy or a monosomy for the affected chromosome. Postzygotic mitotic nondisjunction may then occur as the second event resulting in a rescue of the aneuploidy by either loss of the third chromosome (trisomy rescue) or duplication of a monosomic chromosome (monosomy rescue). ${ }^{4}$ In addition, anaphase lag (the delayed movement

\footnotetext{
${ }^{1}$ Department of Human Genetics, The University of Chicago, Chicago, IL, USA; ${ }^{2}$ Division of Genetics and Genomic Medicine, Department of Pediatrics, Washington University School of Medicine, St. Louis, MO, USA; ${ }^{3}$ Department of Pathology and Laboratory Medicine, Cleveland Clinic, Cleveland, OH, USA; ${ }^{4}$ Department of Pediatrics, Michigan Medical Genetics Laboratories, University of Michigan, Ann Arbor, MI, USA; ${ }^{5}$ Department of Pathology, Duke University, Durham, NC, USA; ${ }^{6}$ Department of Pathology and Laboratory Medicine, Children's Hospital Los Angeles, Keck School of Medicine, University of Southern California, Los Angeles, CA, USA; ${ }^{7}$ American College of Medical Genetics and Genomics, Bethesda, MD, USA. Correspondence: ACMG (documents@acmg.net)

The Board of Directors of the American College of Medical Genetics and Genomics approved this position statement on 24 February 2020.
} 


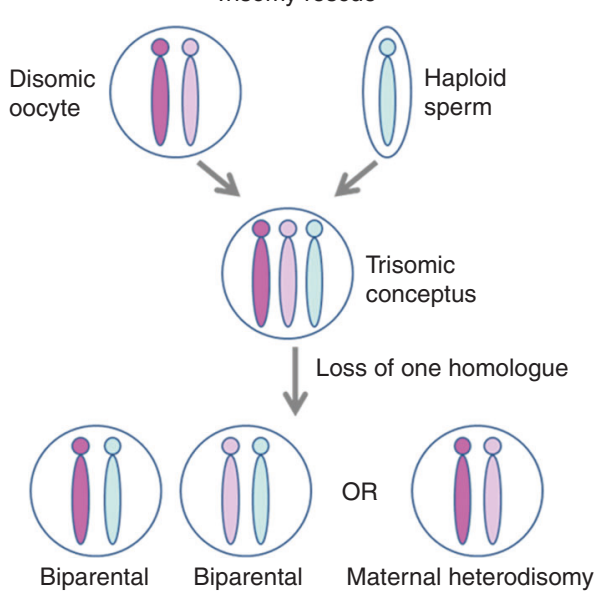

b

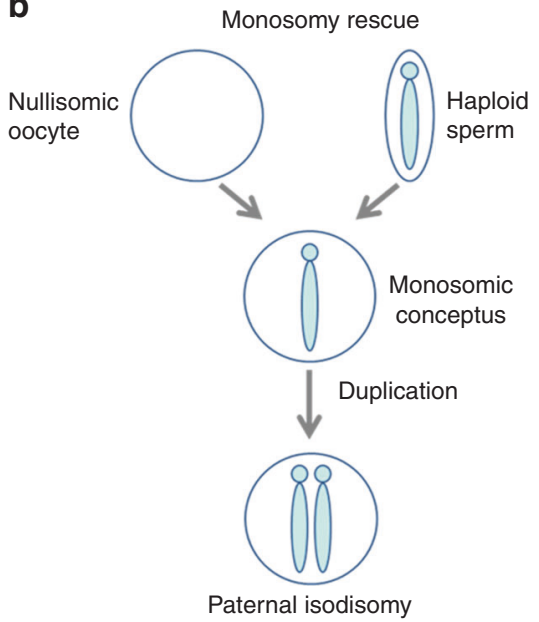

C

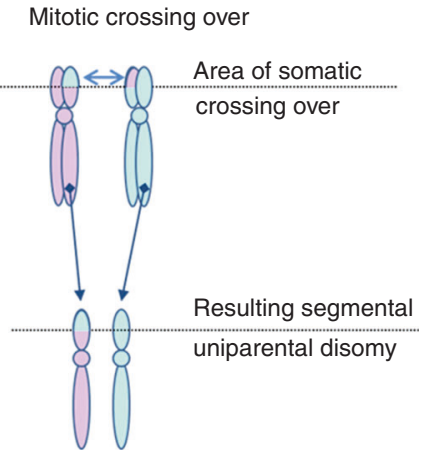

Fig. 1 Common mechanisms resulting in uniparental disomy (UPD). (a) Trisomy rescue mechanism. The example shows maternal nondisjunction in meiosis I resulting in a disomic oocyte. Upon fertilization with a normal sperm this produces a trisomic conceptus. Subsequent trisomy rescue theoretically results in UPD in one-third of the cases. (b) Monosomy rescue mechanism. The example shows maternal nondisjunction in meiosis I resulting in a nullisomic oocyte. Fertilization with a normal sperm produces a monosomic conceptus. Duplication of the only copy of the affected chromosome results in paternal isodisomy. (c) Mitotic crossing over. Somatic mitotic crossing over, resulting in mosaic segmental uniparental disomy. Pink shading, maternal chromosome; blue shading, paternal chromosome.
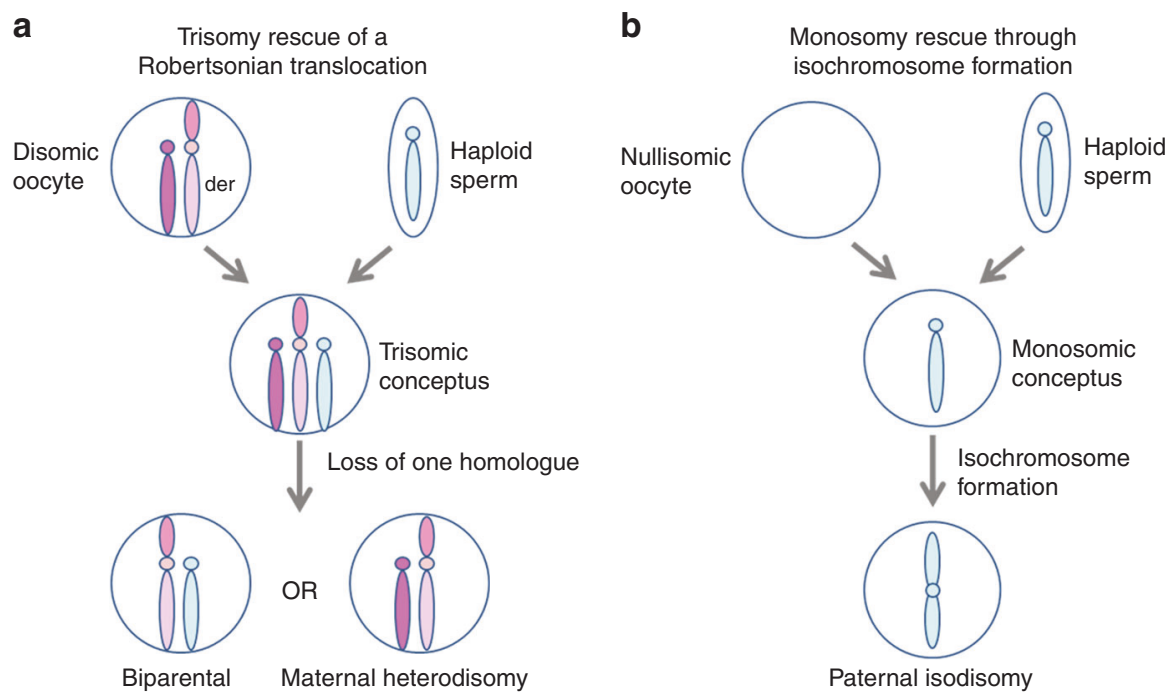

Fig. 2 Common mechanisms resulting in uniparental disomy (UPD) involving acrocentric chromosomal rearrangements. (a) Rescue of a trisomy conceptus from a Robertsonian translocation carrier mother. Disomy in the oocyte results from the presence of the derivative chromosome from the Robertsonian translocation (der) and a normal copy of one of the affected acrocentrics. Fertilization with a normal sperm produces a trisomic conceptus. The rescue results theoretically in UPD in $50 \%$ of the cases. (b) Monosomy rescue of a monosomic conceptus resulting from meiosis I nondisjunction and fertilization of a nullisomic gamete. Duplication (through isochromosome formation) of the only copy of a homologue would result in isodisomy in $100 \%$ of cases. Since the majority of nondisjunction occurs in maternal meiosis, most cases of isochromosomes arising through this mechanism result in paternal isodisomy. Pink shading, maternal chromosome; blue shading, paternal chromosome.

of a chromosome or chromatid during anaphase, leading to the loss of that chromosome in a daughter nucleus) may also be the second event leading to a trisomy rescue. Trisomy rescue with loss of the parental chromosome present in a single copy will result in the inheritance of both homologues of the affected chromosome from one parent or UPD (Fig. 1a). As the majority of nondisjunction occurs in maternal meiosis $\mathrm{I},{ }^{5}$ it is more likely that a trisomy consists of two different maternal chromosomes and one paternal chromosome. Subsequent trisomy rescue through loss of a paternal chromosome will thus give rise to maternal heterodisomy. Meiotic recombination will often result in the presence of one or more regions of homozygosity (ROH) on the affected chromosome, but with retention of heterozygosity around the centromere where recombination is suppressed. Analogously, chromosomes inherited from the same parent in cases of isodisomy due to meiosis II errors often do not show complete homozygosity for all single-nucleotide polymorphism (SNP) markers. Due to meiotic recombination, 
such chromosomes may also have alternating regions of heterozygosity and homozygosity, but in meiosis II errors, there is always homozygosity around the centromere. Postzygotic monosomy rescue (which is more rare than trisomy rescue) will result in complete isodisomy of the same homologue, with no heterodisomic regions (Fig. 1b). There are also cases of mosaic, segmental UPD affecting terminal regions of chromosome arms; they arise as postzygotic events, due to mitotic recombination between chromatids occuring in early embryogenesis (Fig. 1c). This UPD mechanism is responsible for a subset of cases of Beckwith-Wiedemann syndrome (BWS), an imprinting disorder resulting from altered activity of one or more genes in the imprinted gene cluster in the p15.5 region of chromosome 11 (as discussed later). ${ }^{6}$

Other rare mechanisms leading to UPD have been reported and include postfertilization error (via somatic recombination or gene conversion), gamete complementation, somatic replacement of a derivative chromosome, correction of interchange monosomy, and correction of a trisomy resulting in a small supernumerary marker chromosome (sSMC). ${ }^{4}$ UPD has also been observed to result from the presence of structurally abnormal chromosomes, including Robertsonian translocations, isochromosomes, reciprocal translocations, derivative chromosomes, and inversions (Fig. 2).

As mentioned above, for the majority of chromosomes, there is no apparent phenotypic effect from UPD. ${ }^{7}$ However, a few chromosomes contain regions with parent-specific gene expression (imprinting), and UPD of these chromosomes may lead to clinically recognizable consequences. Specific phenotypes have been well documented to date for maternal UPD for chromosomes 7, 11, 14, 15, and 20, and paternal UPD for chromosomes $6,11,14,15$, and 20. For some chromosomes (2 and 16 for example), it is still debated whether UPD has phenotypic effects attributable to imprinting. This uncertainty may be due to the subtle nature of the anomalies (e.g., maternal disomy 16), ${ }^{8}$ conflicting reports in the literature (e.g., maternal disomy 2), ${ }^{9}$ confounding mosaicism (e.g., maternal disomy 2 and 16), or too few cases reported.

The empiric risks for UPD following the observation of prenatal aneuploidy mosaicism for certain chromosomes or a prenatally diagnosed Robertsonian translocation have been reported. The chance that trisomy 15 mosaicism, observed prenatally as confined placental mosaicism on analysis of chorionic villus sampling (CVS), would result in UPD has been estimated to be $11 \%$ to $25 \%{ }^{10-12}$ For prenatally identified Robertsonian translocations (de novo or inherited) between nonhomologous chromosomes (e.g., der[13;14]), the risk of UPD in the translocation carrier fetus is approximately $0.6 \%{ }^{13,14}$ For homologous acrocentric rearrangements, for which the majority are de novo isochromosomes (i.e., chromosomes derived from a duplication of a single parental chromosome), the risk of UPD in the balanced carrier fetus is approximately $66 \%{ }^{13}$

The prevalence of UPD associated with a clinical presentation due to imprinting disorders or recessive diseases ranges from 1 in 3500 to 1 in $5000 .{ }^{15,16}$ Recent data, collected using over four million consented research participants from the personal genetics company 23andMe and 431,094 northern European UK Biobank participants, estimated that UPD for all chromosomes (rather than just chromosomes carrying imprinted regions) occurs with an overall prevalence of 1 in 2000 births. Since the 23andMe database comprises, for the most part, healthy individuals from the general population, this is a more representative estimate of the overall UPD prevalence in the general population. ${ }^{17}$

\section{TESTING METHODOLOGIES}

Evaluation of DNA-based polymorphic markers is the typical approach to investigate UPD. Short tandem repeat (STR) markers are used for most UPD studies. These markers are abundant throughout the genome, many have very high heterozygosity values (a reflection of the allele frequency differences in the population), and they are ideally suited for multiplex polymerase chain reaction (PCR) ${ }^{18-20}$ In addition to the proband's DNA sample, a sample from both parents is required to delineate the parental origin of the detected STR alleles. If both parents are not available, testing can be performed using one parent; however, in some cases, testing of a single parent may not completely rule out heterodisomy of the other parent. Multiple markers should be tested for each chromosome of interest.

It is strongly recommended that at least two fully informative loci, showing either UPD or biparental inheritance, should be identified for diagnostic reporting. ${ }^{21}$ Multiple, highly polymorphic STR markers across each chromosome of interest should be selected based on their informativity and genomic location. ${ }^{22-24}$ However, there are limitations to this technology in the detection of UPD in samples with somatic mosaicism, segmental UPD, and tissuespecific UPD. Diagnostic reporting should follow the International System for Human Cytogenomic Nomenclature (ISCN) 2016 guidelines: uniparental disomy is abbreviated as "upd" (lowercase), followed by the chromosome in parentheses, and then the parental origin. ${ }^{25}$

In clinical practice, UPD cases may be ascertained through testing for copy-number abnormalities using chromosomal microarray (CMA) platforms that have SNP probes. CMA can easily identify whole-chromosome isodisomy based on obligatory presence of extensive $\mathrm{ROH}$ on the affected chromosome including its pericentromeric region, but routine CMA analysis cannot determine the parental origin without testing parental samples. Furthermore, isodisomy constitutes only a small subset of UPD cases. Whole-chromosome heterodisomy is more common, and can be suspected in CMA testing based on its frequent association with $\mathrm{ROH}$ on the affected chromosome, generated by meiotic crossovers during meiosis in parental gametogenesis. ${ }^{26}$ However, it has been shown that approximately one-third of all cases of molecularly confirmed UPD do not show extended $\mathrm{ROH}$ and are not detectable by $\mathrm{CMA}^{27}$ Even when present, $\mathrm{ROH}$ associated with heterodisomy varies in size, and overlap in the sizes has been demonstrated between $\mathrm{ROH}$ that was 
associated with UPD, and $\mathrm{ROH}$ that occurred due to chance (identity by state) or parental consanguinity (identity by descent). ${ }^{27}$ Laboratories should define size thresholds and other criteria for reporting $\mathrm{ROH}$ and recommending followup UPD testing. Terminal $\mathrm{ROH}$ has been shown to rarely occur in non-UPD cases, and may warrant reporting even when it is relatively small $(5 \mathrm{Mb})$; for interstitial $\mathrm{ROH}$, it has been proposed that larger size thresholds $(15-20 \mathrm{Mb})$ may provide sufficient sensitivity without resulting in high falsepositive rates. ${ }^{27}$ Follow-up testing is indicated primarily if the $\mathrm{ROH}$ region is confined to one chromosome, involves one of the chromosomes that contain imprinted regions, and UPD for that chromosome is expected to result in an abnormal phenotype. Importantly, the $\mathrm{ROH}$ identified by CMA does not have to (and typically does not) overlap with the imprinted region on the affected chromosome. Regardless of where it localizes on the chromosome, the $\mathrm{ROH}$ in this situation functions as a marker of potential wholechromosome UPD, which if confirmed will be responsible for the imprinting disorder in the patient. The presence of extended $\mathrm{ROH}$ on multiple chromosomes most commonly indicates a familial relationship between the proband's parents (consanguinity); recommendations for documenting suspected consanguinity as an incidental finding of genomic testing have been described elsewhere. ${ }^{28}$

Computational algorithms can also be employed to detect UPD through analysis of SNP distribution from trio genotype data in the context of exome or genome sequencing. ${ }^{29,30}$ These tools have been used to identify whole-chromosome UPD and segmental UPD greater than $10 \mathrm{Mb}$ from exome data. ${ }^{31}$ Since heterozygous deletions can masquerade as segmental UPD, follow-up testing has to be performed to distinguish between these two abnormalities. In most clinical laboratories, evaluation for UPD is not routinely incorporated into clinical exome or genome sequencing assays. However, if UPD is detected during analysis, it can be reported as a secondary finding with a recommendation to confirm the finding with a clinically validated assay, for patients who consented to receive secondary findings.

Since these methodologies can potentially uncover misattributed relationships, this possibility should be addressed during the consent or pretest counseling process. Laboratories should develop a process for appropriate follow-up when misattributed relationships are suspected based on the UPD testing results.

In addition to techniques that directly detect UPD, multiple technologies are used in the diagnosis of imprinting disorders that can be caused by UPD for certain chromosomes but can also have other etiologies. Some of these techniques, like methylation-specific PCR (MS-PCR) ${ }^{32}$ and methylationspecific multiplex ligation-dependent probe amplification (MS-MLPA) $)^{33}$ are based on interrogation of the methylation status of so-called differentially methylated regions (DMRs) or imprinting centers within the larger (typically several megabases in size) areas of chromosomes containing imprinted genes. DMRs have different methylation status on the maternal and the paternal homologue; they have been mapped, sequenced, and functionally characterized within well-known clinically significant imprinted chromosomal regions, and are thought to play a role in establishing and maintaining the parent-of-origin specific expression of the surrounding imprinted genes. ${ }^{34}$ MS-PCR and MS-MLPA assays are designed to distinguish between the normal methylation profile of the DMRs on the chromosome of interest and the abnormal profile associated with an imprinting disorder, without the need for parental samples. However, neither technique can distinguish between UPD and imprinting defects. Thus, STR marker analysis is required to determine if UPD is the cause of the observed aberrant methylation pattern.

\section{CHROMOSOMES OF CLINICAL RELEVANCE}

UPD for any chromosome is associated with an increased risk for a recessive disorder, since it can result in an affected child when only one parent is a carrier of a pathogenic variant. This is true even for cases of heterodisomy, due to isodisomic regions generated through meiotic crossovers during gametogenesis in the parent. Individuals at risk for a recessive disorder due to a UPD event may be ascertained by CMA testing. Further evaluation for a recessive disorder may be recommended if there is a concern for UPD based on large $\mathrm{ROH}$ on a single chromosome detected by CMA testing, in particular if there is also a clinical suspicion for a recessive condition based on a physical examination or results of other auxiliary diagnostic studies. More often the occurrence of a recessive disorder due to UPD is identified when sequencing based testing shows homozygosity for a pathogenic variant for which only one parent is a carrier.

UPD for specific chromosomes results in abnormal phenotypes shown to be caused by imprinting.

\section{Paternal UPD6 and transient neonatal diabetes mellitus} Transient neonatal diabetes mellitus (TNDM, MIM 601410) is a rare but well recognized type of diabetes caused by overexpression of the imprinted loci PLAGL1 and HYMAI at chromosome 6q24.2. ${ }^{35,36}$ Partial or complete paternal UPD6 including PLAGL1 and HYMAI has been reported in approximately $40 \%$ of cases of TNDM. ${ }^{37}$ The finding of macroglossia or other congenital anomalies in addition to TNDM is a strong indicator to suspect UPD. ${ }^{37}$ The majority of paternal UPD6 is isodisomic and therefore affected individuals are at increased risk for rare autosomal recessive disorders including HFE-associated hereditary hemochromatosis (MIM 235200), methylmalonic acidemia (MIM 251000), and congenital adrenal hyperplasia caused by 21-hydroxylase deficiency (MIM 201910). ${ }^{37}$

\section{Maternal UPD7 and Russell-Silver syndrome}

Russell-Silver syndrome (RSS, MIM 180860) is characterized by prenatal and postnatal poor growth, relative macrocephaly, 
and limb, body, and/or facial asymmetry. Complete and partial maternal UPD7 accounts for $\sim 7-10 \%$ of patients with RSS. $^{38-42}$ Partial UPD7 due to segmental maternal UPD restricted to the long arm of chromosome 7 , which results in hypermethylation of the imprinting center of the MEST gene within 7q32.2, was reported in several RSS patients. ${ }^{43}$ Maternal UPD7 isodisomy and maternal heterodisomy have been reported in RSS patients. ${ }^{41,44}$ Mosaic maternal segmental UPD of $7 q$ has also been reported in some cases. ${ }^{45,46}$

\section{Paternal UPD11 and Beckwith-Wiedemann syndrome}

Beckwith-Wiedemann syndrome (BWS, MIM 130650) is a congenital overgrowth disorder with a predisposition to tumorigenesis. The disorder is caused by abnormalities within the two differentially methylated regions (DMRs) on the short arm of chromosome 11: imprinting center 1 (IC1), which regulates the expression of $H 19$ and IGF2, and imprinting center 2 (IC2), which regulates the expression of CDKN1C, KCNQ1, and KCNQ10T1. The common causes of BWS are methylation abnormalities affecting the imprinting centers. ${ }^{47}$ Segmental paternal UPD of 11 p15 occurs in about $20 \%$ of BWS patients and results in biallelic expression of the normally paternally expressed IGF2 (in IC1 region), encoding a potent fetal growth factor. ${ }^{48}$ The UPD appears to consistently arise from a somatic recombination event resulting in paternal isodisomy (Fig. 1c). It has been hypothesized that nonmosaic whole-chromosome UPD11 may be lethal, and in fact, mosaicism is present in the majority of the cases, confirming the postzygotic origin of this UPD. ${ }^{6}$ The detection of nonmosaic $\mathrm{ROH}$ involving chromosome 11 in a prenatal setting may be concerning about the high likelihood of fetus lethality.

\section{Maternal UPD11 and Russell-Silver syndrome}

Maternal UPD of chromosome 11 has been rarely described as the cause of isolated cases of RSS. ${ }^{49-52}$ Chromosome 11p15-related RSS is associated primarily with hypomethylation of IC1; this leads to biallelic expression of H19 and biallelic silencing of IGF2, resulting in growth restriction. ${ }^{52}$ Fewer than ten cases of RSS due to maternal UPD11 have been reported to date. ${ }^{49-51}$ The phenotype is indistinguishable from RSS cases caused by other mechanisms, and includes growth restriction, asymmetry, and relative macrocephaly; all reported cases showed mosaicism, consistent with the postzygotic origin of this UPD. ${ }^{50,51}$ It has been hypothesized that mosaic maternal UPD11 may be a more common cause of RSS than is currently appreciated. ${ }^{51}$ However, this abnormality is very difficult to detect, either due to low levels of mosaicism or because it frequently involves tissues other than the peripheral blood cells most often sampled for testing. 50,51

\section{Maternal UPD14 and Temple syndrome}

Temple syndrome (TS, MIM 616222) is characterized by preand postnatal poor growth, mild developmental delay, hypotonia, hyperextensible joints, small hands and feet, truncal obesity, precocious or early onset of puberty, and adult short stature. ${ }^{53}$ Maternal UPD14 is the most widely recognized cause of TS; it results in loss of expression of all paternally expressed genes (DLK1, RTL1, and DIO3) and overexpression of maternally expressed genes (noncoding RNAs GTL2/MEG3, MEG8, RTL1as, and additional microRNAs [miRNAs] and small nucleolar RNAs [snoRNAs]) at chromosome $14 \mathrm{q} 32.2 .^{54,55}$ In rare cases, maternal UPD14 has been reported in association with mosaicism, ${ }^{56}$ Robertsonian translocations, ${ }^{57}$ and sSMC. ${ }^{58}$

\section{Paternal UPD14 and Kagami-Ogata syndrome}

Kagami-Ogata syndrome (KOS, MIM 608149) is characterized by a severe phenotype with polyhydramnios, large omphalocele, thoracic dysplasia (coat-hanger sign on X-rays) with respiratory failure, abdominal wall defects, poor growth, developmental delay, and facial abnormalities including full cheeks and protruding philtrum. ${ }^{59}$ Paternal UPD14 accounts for approximately two-thirds of KOS patients; the remaining cases have been associated with microdeletions affecting the maternal chromosome 14 and epigenetic defects. $^{59,60}$ Excessive RTL1 expression and absent $M E G$ expression in the q32.2 region of chromosome 14 constitute the primary underlying factors for the phenotypic abnormalities in these patients. ${ }^{59,60}$

\section{Maternal UPD15 and Prader-Willi syndrome}

Prader-Willi syndrome (PWS, MIM 176270) is characterized by neonatal hypotonia and poor suck with failure to thrive, developmental delay and/or intellectual disability, childhoodonset obesity, short stature, hypogonadism, and behavior problems. Maternal UPD15 is the second most common finding in patients with PWS and accounts for approximately $20-30 \%$ of the cases. ${ }^{61}$ Patients with maternal UPD15 lack the activity of imprinted, paternally expressed genes in the 15q11.2-q13 region (MKRN3, MAGEL2, NDN, SNURF$S N R P N$, and several snoRNA genes) and have overexpression of maternally expressed genes (UBE3A and ATP10C). ${ }^{62-64}$ Maternal nondisjunction events resulting in UPD15-PWS are associated with meiosis I errors, with rare cases originating from meiosis II or due to postzygotic errors. ${ }^{65}$ Maternal UPD15 has been found in association with Robertsonian translocations, mosaicism, isochromosomes, and sSMC involving chromosome 15.

\section{Paternal UPD15 and Angelman syndrome}

Angelman syndrome (AS, MIM 105830) is associated with severe intellectual disability with absent speech, ataxic movements and gait, increased tone after infancy, microcephaly, seizures, and a happy disposition with paroxysmal laughter. Paternal UPD15 accounts for approximately 3-7\% of AS cases. Patients with paternal UPD15 lack the activity of the maternally expressed UBE3A and ATP10C genes, within the 15q11.2-q13 region. Imprinted $U B E 3 A$ expression is restricted to brain cells, 
and the lack of its expression from the maternal chromosome 15 is considered to be the major cause of the disease phenotype. ${ }^{66}$ Generally, paternal UPD15 cases associated with AS reflect isodisomic UPD as a consequence of a postzygotic mitotic error, though cases due to errors at meiosis II (MII) also occur. ${ }^{65}$ Paternal UPD15 may be rarely due to a parental Robertsonian translocation or isochromosomes. ${ }^{67,68}$

\section{Maternal UPD20 and Mulchandani-Bhoj-Conlin syndrome} Maternal UPD of chromosome 20 (Mulchandani-Bhoj-Conlin syndrome, MIM 617352), without evidence of trisomy 20 mosaicism, is a rare disorder with fewer than 20 patients reported in the literature. The condition is characterized by intrauterine and postnatal poor growth and prominent feeding difficulties with failure to thrive. Most patients do not have dysmorphic features, congenital abnormalities, or major developmental delay. There is a significant phenotypic overlap with RSS and other conditions that predominantly exhibit pre- and postnatal poor growth and short stature. Unexpectedly, in the majority of published cases, CMA testing had genotyping patterns suggesting UPD due to meiosis II error or postzygotic mitotic error, rather than the typically more common meiosis I nondisjunction. ${ }^{69,70}$

\section{Paternal UPD20}

Paternal UPD of chromosome 20 results in pseudohypoparathyroidism type 1b (PHP1B, MIM 603233), which is characterized by resistance to parathyroid hormone in kidneys and presents as hypocalcemia, hyperphosphatemia, and abnormally high parathyroid hormone levels. The condition typically results from deletions in the DMR of the GNAS locus on $20 \mathrm{q}$, or a deletion in the STX gene, which acts as a long-range control element of methylation at the GNAS locus. These defects result in the absence of expression of the maternal Gs- $\alpha$ isoform in renal tissues. Rare cases where PHP1B was caused by paternal UPD20 have been reported. ${ }^{71}$

\section{INDICATIONS FOR POSTNATAL UPD TESTING}

Clinicians may order postnatal UPD testing to confirm a clinical suspicion of a diagnosis based on a constellation of clinical and physical findings. Confirmation of a diagnosis is important to discuss recurrence risk with the family as well as the clinical course and the prognosis of the condition.

Recommendations for postnatal UPD testing include:

1. Patients evaluated for developmental delay/intellectual disability with or without congenital anomalies and found to have a familial or de novo balanced Robertsonian translocation involving chromosome 14 or $15 .^{72}$

2. Patients evaluated for developmental delay/intellectual disability with or without congenital anomalies and found to have a supernumerary structurally abnormal chromosome derived from chromosome 14 or $15 .^{73}$

3. Patients with homozygosity for a pathogenic variant causing an autosomal recessive disorder when only one parent is a carrier for that variant, in the absence of other explanations such as intragenic deletion and misattributed relationships. ${ }^{74}$

4. Patients with TNDM and hypomethylation within the 6q24 DMR region. Testing for UPD6 can be ordered sequentially or simultaneously with MS-MLPA. ${ }^{37,75}$

5. Patients with clinical suspicion for RSS. Testing for UPD7 can be ordered sequentially or simultaneously with methylation testing of the IC1 on chromosome $11 \mathrm{p} 15 .^{52,76,77}$

6. Patients with BWS found to have loss of methylation at IC2 and gain of methylation at IC1 at 11p15. It should be noted that first-line molecular testing for BWS should include DNA methylation analysis of IC1 and IC2. ${ }^{23}$

7. Patients with clinical findings and physical features suggestive of maternal or paternal UPD14. The UPD testing can be ordered sequentially to or simultaneously with the methylation testing. ${ }^{72,73}$

8. Patients with PWS or AS with abnormal methylation studies (other than MS-MLPA) who have normal karyotype and CMA results. ${ }^{78}$ Although large $\mathrm{ROH}$ observed by CMA testing is highly suspicious for UPD, it requires confirmation using other methods. It is important to keep in mind that DNA methylation analysis is the first-line testing for PWS and AS. ${ }^{78}$

9. Patients with PHP1B who have abnormal methylation studies of the DMRs at the GNAS complex locus with normal karyotype and CMA results. In addition, patients with poor growth and feeding difficulties found to have $\mathrm{ROH}$ for chromosome 20 on SNP array.

10. Female patients who present with unexplained severe manifestations of X-linked conditions and who are found to have homozygosity for a pathogenic variant in an Xlinked gene. ${ }^{74}$

11. Male patients with unexplained father-to-son transmission of an X-linked disorder. ${ }^{3}$

\section{INDICATIONS FOR PRENATAL UPD TESTING} Clinicians may order prenatal UPD testing when other prenatal studies, performed for advanced maternal age or because of a known familial chromosomal aberration, raise concerns for UPD. UPD analysis may also be used to confirm a clinical suspicion of a diagnosis based on a constellation of prenatal ultrasonographic findings. Confirmation of a diagnosis is important in order to discuss the clinical course and the prognosis of the condition and can be used later for genetic counseling purposes when the recurrence risk is discussed with the family. Timing and turnaround time are critical compared with postnatal testing because of the limited time available for decision making.

Recommendations for prenatal UPD testing include:

1. Level II or level III mosaicism for trisomy or monosomy of chromosomes $6,7,11,14,15$, or 20 in amniocentesis or CVS. ${ }^{79}$ 
2. Level II or level III mosaicism for trisomy or monosomy of chromosomes $6,7,11,14,15$, or 20 in CVS followed by normal karyotype in amniocentesis. ${ }^{80}$

(Note: Level II (multiple cell pseudomosaicism) ${ }^{81}$ : same abnormality observed in two or more cells (flask method) or in two or more cells from one or more colonies (in situ) in the same culture. ${ }^{82}$ Level III (true mosaicism $)^{81}$ : two or more cells with the same abnormality observed in two or more independent cultures.)

3. In the context of preimplantation genetic screening (PGS), a transfer of mosaic embryos with trisomy or monosomy of chromosomes $6,7,11,14,15$, or 20 should be followed by prenatal studies including UPD testing. ${ }^{83-85}$ Since embryos with a completely normal karyotype are rare in the context of PGS, detection of mosaic aneuploidy does not prohibit transfer. As discussed under UPD mechanisms, one of the processes that leads to mosaicism may involve an initially abnormal conceptus, typically due to a meiotic error, with a subsequent rescue mechanism via a mitotic event generating a normal cell line. Timing of the rescue will determine the distribution of the normal and the abnormal cell lines in the fetus and the placenta. For embryos with mosaicism, rescue may generate a fetus with a normal karyotype but with a risk for UPD.

4. Prenatal imaging anomalies consistent with a UPD phenotype. The classic example is the pathognomonic coat-hanger sign in paternal UPD $14 .{ }^{86}$ Omphalocele, macroglossia, visceromegaly, enlarged adrenals, or macrosomia with no obvious mechanism are also typical prenatal findings in BWS. ${ }^{87}$ Native amniotic fluid is the preferred tissue for UPD11 testing but the degree of mosaicism may not correlate with the true mosaicism in the fetus and therefore the prediction of postnatal phenotypic outcome is challenging. Furthermore, in the case of negative results the presence of mosaic UPD cannot be excluded. ${ }^{80}$ On the other hand, fetal growth restriction can be considered as a relative indication to test for RSS or Mulchandani-Bhoj-Conlin syndrome but this finding is relatively common and its presence alone should not be an indication.

5. Familial or de novo balanced Robertsonian translocation or isochromosome involving chromosome 14 or 15 based on CVS or amniocentesis. ${ }^{72,88,89}$ Both familial and de novo translocations are associated with an increased risk for UPD.

6. De novo sSMC with no apparent euchromatic material in the fetus. ${ }^{90,91}$

7. Non-Robertsonian translocation between an imprinted chromosome with possible 3:1 disjunction that can lead to trisomy or monosomy rescue or gamete complementation. Although every chromosome abnormality that increases the occurrence of nondisjunction in theory would increase the risk for UPD of the chromosomes involved, there are only very few cases reported. ${ }^{38,92}$

\section{SUMMARY OF CLINICAL AND DIAGNOSTIC CONSIDERATIONS}

1. Chromosomes of known clinical relevance for UPD include 6, 7, 11, 14, 15, and 20.

2. UPD testing should be considered for:

a. Evaluation of a subject with clinical, physical, or ultrasonographic features of disorders known to be associated with UPD.

b. Molecular investigation of a condition that does not follow a typical Mendelian inheritance pattern including recessive and $\mathrm{X}$-linked disorders.

c. Prenatal or postnatal identification of a structurally abnormal chromosome 14 or 15 .

d. Prenatal trisomy or monosomy mosaicism of a chromosome known to be associated with a UPD phenotype.

3. Testing should be performed on DNA collected from the child/fetus and at least one parent using polymorphic markers.

4. UPD can be ascertained through analysis of SNP distribution from trio genotype data in the context of exome or genome sequencing. However, unless the UPD analysis is validated by the diagnostic laboratory for clinical use, confirmation by a clinically validated STRbased assay is required. Detection of isodisomic UPD by CMA warrants clinical correlation and further testing to determine parent of origin.

5. Reporting of results includes at least two fully informative markers from each chromosome of interest and uses the current ISCN guidelines. ${ }^{25}$

\section{DISCLOSURE}

D.d.G., C.A., M.K.T., K.L.D., and G.R. all serve as directors in clinical laboratories that perform a breadth of genetic and genomic analyses on a fee-for-service basis. M.S. declares no conflicts of interest.

Publisher's note Springer Nature remains neutral with regard to jurisdictional claims in published maps and institutional affiliations.

\section{REFERENCES}

1. Engel $E$. [A new genetic concept: the uniparental disomy and its potential effect, the isodisomy (author's transl)]. J Genet Hum. 1980;28:11-22.

2. Spence JE, Perciaccante RG, Greig GM, et al. Uniparental disomy as a mechanism for human genetic disease. Am J Hum Genet. 1988:42:217-226.

3. Ferrier RA, Lowry RB, Lemire EG, Stoeber GP, Howard J, Parboosingh JS. Father-to-son transmission of an X-linked gene: a case of paternal sex chromosome heterodisomy. Am J Med Genet A. 2009;149A:2871-2873.

4. Ledbetter DH, Engel E. Uniparental disomy in humans: development of an imprinting map and its implications for prenatal diagnosis. Hum Mol Genet. 1995;4:1757-1764. 
5. Koehler KE, Hawley RS, Sherman S, Hassold T. Recombination and nondisjunction in humans and flies. Hum Mol Genet. 1996:5:1495-1504.

6. Slatter RE, Elliott M, Welham K, et al. Mosaic uniparental disomy in Beckwith-Wiedemann syndrome. J Med Genet. 1994;31:749-753.

7. Kotzot D, Utermann G. Uniparental disomy (UPD) other than 15: phenotypes and bibliography updated. Am J Med Genet A. 2005; 136:287-305.

8. Scheuvens R, Begemann M, Soellner L, et al. Maternal uniparental disomy of chromosome 16 [upd(16)mat]: clinical features are rather caused by (hidden) trisomy 16 mosaicism than by upd(16)mat itself. Clin Genet. 2017:92:45-51.

9. Hansen WF, Bernard LE, Langlois $S$, et al. Maternal uniparental disomy of chromosome 2 and confined placental mosaicism for trisomy 2 in a fetus with intrauterine growth restriction, hypospadias, and oligohydramnios. Prenat Diagn. 1997;17:443-450.

10. Trisomy 15 CPM: probable origins, pregnancy outcome and risk of fetal UPD: European Collaborative Research on Mosaicism in CVS (EUCROMIC). Prenat Diagn. 1999;19:29-35.

11. Christian SL, Smith AC, Macha M, et al. Prenatal diagnosis of uniparental disomy 15 following trisomy 15 mosaicism. Prenat Diagn. 1996;16:323-332.

12. Robinson WP, Langlois S, Schuffenhauer S, et al. Cytogenetic and agedependent risk factors associated with uniparental disomy 15. Prenat Diagn. 1996;16:837-844.

13. Berend SA, Horwitz J, McCaskill C, Shaffer LG. Identification of uniparental disomy following prenatal detection of Robertsonian translocations and isochromosomes. Am J Hum Genet. 2000;66:1787-1793.

14. Silverstein S, Lerer I, Sagi M, Frumkin A, Ben-Neriah Z, Abeliovich D. Uniparental disomy in fetuses diagnosed with balanced Robertsonian translocations: risk estimate. Prenat Diagn. 2002;22:649-651.

15. Liehr T. Cytogenetic contribution to uniparental disomy (UPD). Mol Cytogenet. 2010;3:8.

16. Robinson WP. Mechanisms leading to uniparental disomy and their clinical consequences. Bioessays. 2000;22:452-459.

17. Nakka P, Pattillo Smith S, O'Donnell-Luria AH, et al. Characterization of prevalence and health consequences of uniparental disomy in four million individuals from the general population. Am J Hum Genet. 2019;105:921-932.

18. Shaffer LG, McCaskill C, Adkins K, Hassold TJ. Systematic search for uniparental disomy in early fetal losses: the results and a review of the literature. Am J Med Genet. 1998;79:366-372.

19. Huang $\mathrm{TH}$, Cottingham RW, Ledbetter DH, Zoghbi HY. Genetic mapping of four dinucleotide repeat loci, DXS453, DXS458, DXS454, and DXS424, on the $X$ chromosome using multiplex polymerase chain reaction. Genomics. 1992;13:375-380.

20. Shaffer LG, Overhauser J, Jackson LG, Ledbetter DH. Genetic syndromes and uniparental disomy: a study of 16 cases of Brachmann-de Lange syndrome. Am J Med Genet. 1993;47:383-386.

21. Shaffer LG, Agan N, Goldberg JD, Ledbetter DH, Longshore JW, Cassidy SB. American College of Medical Genetics statement of diagnostic testing for uniparental disomy. Genet Med. 2001:3:206-211.

22. Robinson WP, Christian SL, Kuchinka BD, et al. Somatic segregation errors predominantly contribute to the gain or loss of a paternal chromosome leading to uniparental disomy for chromosome 15. Clin Genet. 2000:57:349-358.

23. Eggermann K, Bliek J, Brioude F, et al. EMQN best practice guidelines for the molecular genetic testing and reporting of chromosome 11 p15 imprinting disorders: Silver-Russell and Beckwith-Wiedemann syndrome. Eur J Hum Genet. 2016;24:1377-1387.

24. Eggermann T, Mergenthaler S, Eggermann K, et al. Identification of interstitial maternal uniparental disomy (UPD) (14) and complete maternal $\operatorname{UPD}(20)$ in a cohort of growth retarded patients. J Med Genet. 2001;38:86-89.

25. McGowan-Jordan J, Simons A, Schmid M. ISCN 2016: an international system for human cytogenetic nomenclature. Basel, Switzerland: Karger; 2016.

26. Kearney HM, Kearney JB, Conlin LK. Diagnostic implications of excessive homozygosity detected by SNP-based microarrays: consanguinity, uniparental disomy, and recessive single-gene mutations. Clin Lab Med. 2011;31:595-613.

27. Hoppman N, Rumilla K, Lauer E, Kearney H, Thorland E. Patterns of homozygosity in patients with uniparental disomy: detection rate and suggested reporting thresholds for SNP microarrays. Genet Med. 2018;20:1522-1527.

28. Rehder CW, David KL, Hirsch B, Toriello HV, Wilson CM, Kearney HM. American College of Medical Genetics and Genomics: standards and guidelines for documenting suspected consanguinity as an incidental finding of genomic testing. Genet Med. 2013;15:150-152.

29. Magi A, Tattini L, Palombo F, et al. H3M2: detection of runs of homozygosity from whole-exome sequencing data. Bioinformatics. 2014;30:2852-2859.

30. King DA, Fitzgerald TW, Miller $R$, et al. A novel method for detecting uniparental disomy from trio genotypes identifies a significant excess in children with developmental disorders. Genome Res. 2014;24:673-687.

31. Bis DM, Schüle R, Reichbauer J, et al. Uniparental disomy determined by whole-exome sequencing in a spectrum of rare motoneuron diseases and ataxias. Mol Genet Genomic Med. 2017;5:280-286.

32. Kubota T, Das S, Christian SL, Baylin SB, Herman JG, Ledbetter DH. Methylation-specific PCR simplifies imprinting analysis. Nat Genet. 1997:16:16-17.

33. Schouten JP, McElgunn CJ, Waaijer R, Zwijnenburg D, Diepvens F, Pals G. Relative quantification of 40 nucleic acid sequences by multiplex ligationdependent probe amplification. Nucleic Acids Res. 2002;30:e57.

34. Kaneko-Ishino T, Kohda T, Ishino F. The regulation and biological significance of genomic imprinting in mammals. J Biochem. 2003;133:699-711.

35. Temple IK, James RS, Crolla JA, et al. An imprinted gene(s) for diabetes? Nat Genet. 1995;9:110-112.

36. Kamiya M, Judson $\mathrm{H}$, Okazaki $\mathrm{Y}$, et al. The cell cycle control gene ZAC/ PLAGL1 is imprinted-a strong candidate gene for transient neonatal diabetes. Hum Mol Genet. 2000;9:453-460.

37. Docherty LE, Kabwama S, Lehmann A, et al. Clinical presentation of $6 q 24$ transient neonatal diabetes mellitus (6q24 TNDM) and genotypephenotype correlation in an international cohort of patients. Diabetologia. 2013;56:758-762.

38. Kotzot D, Schmitt S, Bernasconi F, et al. Uniparental disomy 7 in SilverRussell syndrome and primordial growth retardation. Hum Mol Genet. 1995:4:583-587.

39. Preece MA, Price SM, Davies V, et al. Maternal uniparental disomy 7 in Silver-Russell syndrome. J Med Genet. 1997;34:6-9.

40. Eggermann T, Wollmann HA, Kuner R, et al. Molecular studies in 37 Silver-Russell syndrome patients: frequency and etiology of uniparental disomy. Hum Genet. 1997;100:415-419.

41. Price SM, Stanhope R, Garrett C, Preece MA, Trembath RC. The spectrum of Silver-Russell syndrome: a clinical and molecular genetic study and new diagnostic criteria. J Med Genet. 1999;36:837-842.

42. Eggermann T, Schneider-Rätzke B, Begemann M, Spengler S. Isolated hypermethylation of GRB10 (7p12.2) in a Silver-Russell syndrome patient carrying a 20p13 microdeletion. Clin Genet. 2014;85:399-400.

43. Hannula K, Kere J, Pirinen S, Holmberg C, Lipsanen-Nyman M. Do patients with maternal uniparental disomy for chromosome 7 have a distinct mild Silver-Russell phenotype? J Med Genet. 2001;38:273-278.

44. Bernard LE, Peñaherrera MS, Van Allen MI, et al. Clinical and molecular findings in two patients with Russell-Silver syndrome and UPD7: comparison with non-UPD7 cases. Am J Med Genet. 1999;87:230-236.

45. Reboul MP, Tandonnet O, Biteau N, et al. Mosaic maternal uniparental isodisomy for chromosome 7q21-qter. Clin Genet. 2006;70:207-213.

46. Su J, Wang J, Fan X, et al. Mosaic UPD $(7 q)$ mat in a patient with Silver Russell syndrome. Mol Cytogenet. 2017:10:36

47. Brioude F, Kalish JM, Mussa A, et al. Expert consensus document: Clinical and molecular diagnosis, screening and management of Beckwith-Wiedemann syndrome: an international consensus statement. Nat Rev Endocrinol. 2018;14:229-249.

48. Choufani S, Shuman C, Weksberg R. Beckwith-Wiedemann syndrome. Am J Med Genet C Semin Med Genet. 2010;154C:343-354.

49. Luk HM, Yeung KS, Wong WL, Chung BH, Tong TM, Lo IF. Silver-Russell syndrome in Hong Kong. Hong Kong Med J. 2016;22:526-533.

50. Luk HM, Ivan Lo FM, Sano S, et al. Silver-Russell syndrome in a patient with somatic mosaicism for upd(11)mat identified by buccal cell analysis. Am J Med Genet A. 2016;170:1938-1941.

51. Bullman H, Lever M, Robinson DO, Mackay DJ, Holder SE, Wakeling EL. Mosaic maternal uniparental disomy of chromosome 11 in a patient with Silver-Russell syndrome. J Med Genet. 2008;45:396-399. 
52. Eggermann T, Begemann M, Binder G, Spengler S. Silver-Russell syndrome: genetic basis and molecular genetic testing. Orphanet J Rare Dis. 2010;5:19.

53. Ioannides Y, Lokulo-Sodipe K, Mackay DJ, Davies JH, Temple IK. Temple syndrome: improving the recognition of an underdiagnosed chromosome 14 imprinting disorder: an analysis of 51 published cases. J Med Genet. 2014;51:495-501.

54. da Rocha ST, Edwards CA, Ito M, Ogata T, Ferguson-Smith AC. Genomic imprinting at the mammalian Dlk1-Dio3 domain. Trends Genet. 2008;24:306-316

55. Kagami M, Sekita Y, Nishimura G, et al. Deletions and epimutations affecting the human $14 q 32.2$ imprinted region in individuals with paternal and maternal upd(14)-like phenotypes. Nat Genet. 2008:40:237-242.

56. Yakoreva M, Kahre T, Pajusalu S, et al. A new case of a rare combination of Temple syndrome and mosaic trisomy 14 and a literature review. Mol Syndromol. 2018;9:182-189.

57. Bertini V, Fogli A, Bruno R, et al. Maternal uniparental disomy 14 (Temple syndrome) as a result of a Robertsonian translocation. Mol Syndromol. 2017:8:131-138.

58. Tohyama J, Yamamoto T, Hosoki K, et al. West syndrome associated with mosaic duplication of FOXG1 in a patient with maternal uniparental disomy of chromosome 14. Am J Med Genet A. 2011;155A:2584-2588.

59. Kagami M, Kurosawa K, Miyazaki O, Ishino F, Matsuoka K, Ogata T. Comprehensive clinical studies in 34 patients with molecularly defined UPD(14)pat and related conditions (Kagami-Ogata syndrome). Eur J Hum Genet. 2015;23:1488-1498.

60. Sutton VR, Shaffer LG. Search for imprinted regions on chromosome 14: comparison of maternal and paternal UPD cases with cases of chromosome 14 deletion. Am J Med Genet. 2000;93:381-387.

61. Cassidy SB, Schwartz S, Miller JL, Driscoll DJ. Prader-Willi syndrome. Genet Med. 2012;14:10-26.

62. Chamberlain SJ, Brannan CI. The Prader-Willi syndrome imprinting center activates the paternally expressed murine Ube3a antisense transcript but represses paternal Ube3a. Genomics. 2001;73:316-322.

63. Glenn CC, Saitoh S, Jong MT, et al. Gene structure, DNA methylation, and imprinted expression of the human SNRPN gene. Am J Hum Genet. 1996;58:335-346.

64. Runte M, Hüttenhofer A, Gross S, Kiefmann M, Horsthemke B, Buiting K. The IC-SNURF-SNRPN transcript serves as a host for multiple small nucleolar RNA species and as an antisense RNA for UBE3A. Hum Mol Genet. 2001;10:2687-2700.

65. Fridman C, Koiffmann CP. Origin of uniparental disomy 15 in patients with Prader-Willi or Angelman syndrome. Am J Med Genet. 2000;94:249-253.

66. Buiting K. Prader-Willi syndrome and Angelman syndrome. Am J Med Genet C Semin Med Genet. 2010;154C:365-376.

67. Freeman SB, May KM, Pettay D, Fernhoff PM, Hassold TJ. Paternal uniparental disomy in a child with a balanced 15;15 translocation and Angelman syndrome. Am J Med Genet. 1993;45:625-630.

68. Poyatos D, Guitart M, Gabau E, et al. Severe phenotype in Angelman syndrome resulting from paternal isochromosome 15. J Med Genet. 2002;39:E4.

69. Mulchandani S, Bhoj EJ, Luo M, et al. Maternal uniparental disomy of chromosome 20: a novel imprinting disorder of growth failure. Genet Med. 2016;18:309-315.

70. Kawashima S, Nakamura A, Inoue T, et al. Maternal uniparental disomy for chromosome 20: physical and endocrinological characteristics of five patients. J Clin Endocrinol Metab. 2018;103:2083-2088.

71. Dixit A, Chandler KE, Lever M, et al. Pseudohypoparathyroidism type $1 \mathrm{~b}$ due to paternal uniparental disomy of chromosome 20q. J Clin Endocrinol Metab. 2013;98:E103-108.
72. Yip MY. Uniparental disomy in Robertsonian translocations: strategies for uniparental disomy testing. Transl Pediatr. 2014;3:98-107.

73. Liehr $\mathrm{T}$, Klein $\mathrm{E}$, Mrasek $\mathrm{K}$, et al. Clinical impact of somatic mosaicism in cases with small supernumerary marker chromosomes. Cytogenet Genome Res. 2013;139:158-163.

74. Niida Y, Ozaki M, Shimizu M, Ueno K, Tanaka T. Classification of uniparental isodisomy patterns that cause autosomal recessive disorders: proposed mechanisms of different proportions and parental origin in each pattern. Cytogenet Genome Res. 2018;154:137-146.

75. Alkorta-Aranburu G, Sukhanova M, Carmody D, et al. Improved molecular diagnosis of patients with neonatal diabetes using a combined next-generation sequencing and MS-MLPA approach. J Pediatr Endocrinol Metab. 2016;29:523-531.

76. Eggermann T, Spengler S, Gogiel $M$, Begemann $M$, Elbracht $M$. Epigenetic and genetic diagnosis of Silver-Russell syndrome. Expert Rev Mol Diagn. 2012;12:459-471.

77. Wakeling EL, Brioude F, Lokulo-Sodipe O, et al. Diagnosis and management of Silver-Russell syndrome: first international consensus statement. Nat Rev Endocrinol. 2017;13:105-124.

78. Ramsden SC, Clayton-Smith J, Birch R, Buiting K. Practice guidelines for the molecular analysis of Prader-Willi and Angelman syndromes. BMC Med Genet. 2010;11:70.

79. Eggermann T, Soellner L, Buiting K, Kotzot D. Mosaicism and uniparental disomy in prenatal diagnosis. Trends Mol Med. 2015;21:77-87.

80. Grati FR, Grimi B, Frascoli G, et al. Confirmation of mosaicism and uniparental disomy in amniocytes, after detection of mosaic chromosome abnormalities in chorionic villi. Eur J Hum Genet. 2006;14:282-288.

81. Hsu LY, Kaffe S, Jenkins EC, et al. Proposed guidelines for diagnosis of chromosome mosaicism in amniocytes based on data derived from chromosome mosaicism and pseudomosaicism studies. Prenat Diagn. $1992 ; 12: 555-573$.

82. Winsor EJ, Tomkins DJ, Kalousek D, et al. Cytogenetic aspects of the Canadian early and mid-trimester amniotic fluid trial (CEMAT). Prenat Diagn. 1999;19:620-627.

83. Besser AG, Mounts EL. Counselling considerations for chromosomal mosaicism detected by preimplantation genetic screening. Reprod Biomed Online. 2017;34:369-374.

84. Grati FR, Gallazzi G, Branca L, Maggi F, Simoni G, Yaron Y. An evidencebased scoring system for prioritizing mosaic aneuploid embryos following preimplantation genetic screening. Reprod Biomed Online. 2018;36:442-449.

85. Sachdev NM, Maxwell SM, Besser AG, Grifo JA. Diagnosis and clinical management of embryonic mosaicism. Fertil Steril. 2017;107:6-11.

86. Curtis L, Antonelli E, Vial Y, et al. Prenatal diagnostic indicators of paternal uniparental disomy 14. Prenat Diagn. 2006;26:662-666.

87. Eggermann T, Brioude F, Russo $S$, et al. Prenatal molecular testing for Beckwith-Wiedemann and Silver-Russell syndromes: a challenge for molecular analysis and genetic counseling. Eur J Hum Genet. 2016;24:784-793.

88. Eggermann T, Zerres K. Uniparental disomy and Robertsonian translocations: risk estimation and prenatal testing. Mol Diagn. 2003;7:113-117.

89. Shaffer LG. Risk estimates for uniparental disomy following prenatal detection of a nonhomologous Robertsonian translocation. Prenat Diagn. 2006;26:303-307.

90. Kotzot D. Prenatal testing for uniparental disomy: indications and clinical relevance. Ultrasound Obstet Gynecol. 2008;31:100-105.

91. Liehr T, Ewers $E$, Hamid $A B$, et al. Small supernumerary marker chromosomes and uniparental disomy have a story to tell. J Histochem Cytochem. 2011;59:842-848.

92. Behnecke A, Hinderhofer K, Jauch A, Janssen JW, Moog U. Silver-Russell syndrome due to maternal uniparental disomy 7 and a familial reciprocal translocation t(7;13). Clin Genet. 2012;82:494-498. 